University of New Hampshire

University of New Hampshire Scholars' Repository

Health Management and Policy Scholarship

Health Management and Policy

6-2004

\title{
Effects of a Tailored Follow-Up Intervention on Health Behaviors, Beliefs, and Attitudes
}

\author{
Alissa D. Jacobs \\ University of North Carolina at Chapel Hill \\ Alice S. Ammerman \\ University of North Carolina at Chapel Hill \\ Susan T. Ennett \\ University of North Carolina at Chapel Hill \\ Marci K. Campbell \\ University of North Carolina at Chapel Hill \\ Katherine W. Tawney \\ University of North Carolina at Chapel Hill \\ Follow this and additional works at: https://scholars.unh.edu/hmp_facpub \\ Sre next page for additional authors \\ Part of the Community Health and Preventive Medicine Commons, Other Medicine and Health \\ Sciences Commons, Public Health Education and Promotion Commons, and the Women's Health \\ Commons
}

\section{Recommended Citation}

Jacobs, Alissa D.; Ammerman, Alice S.; Ennett, Susan T.; Campbell, Marci K.; Tawney, Katherine W.; Aytur, Semra; Marshall, Stephen W.; Will, Julie C.; and Rosamond, Wayne D., "Effects of a Tailored Follow-Up Intervention on Health Behaviors, Beliefs, and Attitudes" (2004). Journal of Women's Health. 7.

https://scholars.unh.edu/hmp_facpub/7

This Article is brought to you for free and open access by the Health Management and Policy at University of New Hampshire Scholars' Repository. It has been accepted for inclusion in Health Management and Policy Scholarship by an authorized administrator of University of New Hampshire Scholars' Repository. For more information, please contact Scholarly.Communication@unh.edu. 


\section{Authors}

Alissa D. Jacobs, Alice S. Ammerman, Susan T. Ennett, Marci K. Campbell, Katherine W. Tawney, Semra Aytur, Stephen W. Marshall, Julie C. Will, and Wayne D. Rosamond 


\title{
Effects of a Tailored Follow-Up Intervention on Health Behaviors, Beliefs, and Attitudes
}

\author{
ALISSA D. JACOBS, Ph.D., R.D., ${ }^{1}$ ALICE S. AMMERMAN, Dr.P.H., R.D., ${ }^{1}$ \\ SUSAN T. ENNETT, Ph.D., ${ }^{2}$ MARCI K. CAMPBELL, Ph.D., M.P.H., R.D., ${ }^{1}$ \\ KATHERINE W. TAWNEY, Ph.D., ${ }^{3}$ SEMRA A. AYTUR, M.S., ${ }^{4}$ \\ STEPHEN W. MARSHALL, Ph.D., ${ }^{4}$ JULIE C. WILL, Ph.D., ${ }^{5}$ \\ and WAYNE D. ROSAMOND, Ph.D. ${ }^{4}$
}

\begin{abstract}
Background: The high rates of relapse that tend to occur after short-term behavioral interventions indicate the need for maintenance programs that promote long-term adherence to new behavior patterns. Computer-tailored health messages that are mailed to participants or given in brief telephone calls offer an innovative and time-efficient alternative to ongoing face-to-face contact with healthcare providers.

Methods: Following a 1-year behavior change program, 22 North Carolina health departments were randomly assigned to a follow-up intervention or control condition. Data were collected from 1999 to 2001 by telephone-administered surveys at preintervention and postintervention for 511 low-income, midlife adult women enrolled in the Well-Integrated Screening and Evaluation for Women Across the Nation (WISEWOMAN) program at local North Carolina health departments. During the year after the behavior change program, intervention participants were mailed six sets of computer-tailored health messages and received two computer-tailored telephone counseling sessions. Main outcomes of dietary and physical activity behaviors, beliefs, and attitudes were measured.

Results: Intervention participants were more likely to move forward into more advanced stages of physical activity change $(p=0.02)$; control participants were more likely to increase their level of dietary social support at follow-up $(p=0.05)$. Both groups maintained low levels of reported saturated fat and cholesterol intake at follow-up. No changes were seen in physical activity in either group.

Conclusions: Mailed computer-tailored health messages and telephone counseling calls favorably modified forward physical activity stage movement but did not appreciably affect any other psychosocial or behavioral outcomes.
\end{abstract}

\footnotetext{
${ }^{1}$ Department of Nutrition, ${ }^{2}$ Department of Health Behavior and Health Education, ${ }^{3}$ Department of Nephrology and Hypertension, and ${ }^{4}$ Department of Epidemiology, University of North Carolina at Chapel Hill, Chapel Hill, North Carolina.

${ }^{5}$ Centers for Disease Control and Prevention, Division of Nutrition and Physical Activity, Atlanta, Georgia.

Funding for this research was provided by a grant from the Centers for Disease Control and Prevention through a subcontract with the North Carolina State Department of Health and Human Services.
} 


\section{INTRODUCTION}

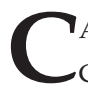
ARdiovascular disease (CVD) is the LEading CAUSE of death among women in the United States, ${ }^{1}$ and mortality rates are particularly high among women of low socioeconomic status or from ethnic minority groups. ${ }^{2-4}$ Lifestyle-related risk factors, such as an unhealthy diet and physical inactivity, are important causes of $\mathrm{CVD}^{5-7}$ and are key targets for prevention. Although intervention programs have been successful in promoting short-term adherence to dietary and physical activity regimens for health promotion, ${ }^{8-11}$ few result in long-lasting change. ${ }^{12-14}$ Indeed, most people who succeed in making changes revert back to less healthy habits within 6-12 months. ${ }^{13}$ Given that sustained changes can lead to substantial reductions in morbidity and mortality, ${ }^{15,16}$ there is a crucial need for maintenance programs aimed at preventing relapse.

Of the studies reporting follow-up contact during the posttreatment period, promising results have been obtained from multifaceted maintenance programs that combine ongoing contact with other strategies, such as relapse prevention training. ${ }^{17-26}$ Few of these studies, however, have targeted low-income, middle-aged women from ethnic minority groups, and even fewer are feasible in public health settings.

A primary means by which states provide healthcare to low-income people is through health departments, but frequently health department staff lack the time and resources to provide preventive health services. ${ }^{27}$ Computer-tailored mail and telephone contacts are a promising means of providing ongoing personalized intervention to large numbers of people. Mail and telephone interventions have been effective in promoting sustained changes in dietary intake $\mathrm{e}^{28-30}$ and physical activity. ${ }^{17-19,26,30-32}$ Moreover, some studies suggest that messages tailored to a person's specific needs and concerns are more effective in promoting changes in diet ${ }^{33-35}$ and physical activity ${ }^{36-39}$ than are standard, nontailored materials.

What had yet to be tested, but offered a promising approach to long-term behavior change, was whether computer-tailored, mailed health messages and brief telephone calls from healthcare providers could promote long-term adherence to positive dietary and physical activity behaviors among people who had participated in a behavior change program. We tested the hypothesis that providing ongoing personalized health informa- tion to midlife, low-income women after they had finished a CVD risk reduction program would promote greater improvements or maintenance of dietary and physical activity behaviors, beliefs, and attitudes relative to a usual care group.

\section{MATERIALS AND METHODS}

Study participants were 511 women who participated in the North Carolina Well-Integrated Screening and Evaluation for Women Across the Nation (WISEWOMAN) nutrition and physical activity program from January through December 1998. The NC WISEWOMAN project was a nonrandomized group-assigned intervention established to demonstrate the feasibility and effectiveness of offering CVD screening and patient education to low-income women, aged 50-64 years, at local health departments. ${ }^{40}$ NC WISEWOMAN consisted of a pilot phase (1995-1997), an initial intervention phase (1998-1999), and a maintenance intervention phase (1999-2001).

\section{Phase One: Pilot study}

In the pilot study, women in the enhanced intervention (EI) received three counseling sessions using a structured assessment and intervention program called New Leaf . . . Choices for Healthy Living. ${ }^{41}$ Results showed that EI participants experienced significant reductions in reported dietary fat and cholesterol intake $(p=0.001)$ compared with essentially no change in women in the minimum intervention (MI) (i.e., usual health department services). ${ }^{42}$ Improvements in total and high-density lipoprotein (HDL) cholesterol, diastolic blood pressure (DBP), body mass index (BMI), and physical activity also were observed but did not differ significantly between groups. ${ }^{42}$ The pilot study results are described in more detail elsewhere. ${ }^{42}$

\section{Phase Two: Initial intervention}

Thirty-three health departments participated in the second phase of NC WISEWOMAN (19981999). Of these, 11 were assigned to MI and 22 to EI. Briefly, in MI health departments, participants at high risk for CVD were given the health department's usual counseling and referred by staff members according to criteria provided by the state health department. Using the New Leaf program, ${ }^{41}$ the EI health departments gave at-risk par- 
ticipants three 30-minute counseling sessions on diet and physical activity over 6 months.

At baseline, $88 \%(n=2061)$ of women screened for CVD risk factors were at elevated risk and enrolled in the intervention $(n=1376$ in EI and $n=$ 685 in MI) ${ }^{43}$ At the 12-month follow-up, 37\% $(n=$ 772) of at-risk women returned to the health departments for rescreening. ${ }^{43}$ Of the EI women $(n=484)$ seen at both baseline and 12-month follow-up, $65 \%(n=314)$ attended at least two intervention sessions. ${ }^{43}$

The analyses on change in risk factors were completed on a subset of participants and restricted to women in the EI group who attended two or more intervention sessions $(n=314)$ or women in the MI group who were enrolled prior to April $1999(n=276)$. On the basis of brief single-item screeners, findings suggest that improvements in self-assessed diet and physical activity were greater in the EI group than in the MI group. From baseline to 12-month follow-up, the percentage of women who reported consuming a "very" or "pretty much" heart-healthy diet increased from $43 \%$ to $67 \%$ in the EI group vs. $50 \%$ to $57 \%$ in the MI group. ${ }^{43}$ Similarly, the percentage of women reporting $>30$ minutes of moderate activity on most days increased from $60 \%$ to $68 \%$ in the EI group and $62 \%$ to $66 \%$ in the MI group. ${ }^{43}$ Because of the significant loss to followup, however, no formal statistical tests or adjustments for clustering design were computed because the data were not considered valid. Accordingly, in Phase Three we chose to conservatively define "maintenance" as maintenance of behaviors that may have been present before the initial intervention (rather than maintenance of behaviors resulting from the intervention).

\section{Phase Three: Maintenance study}

After the initial intervention, the 22 EI health departments were randomly assigned to maintenance special intervention (MSI) or maintenance usual care (MUC) (Fig. 1). The eligible study population for the maintenance study consisted of 909 women who enrolled in the EI between January and December 1998. Of these, 511 completed the baseline maintenance survey and were enrolled in the follow-up program. During the 1year maintenance program, MUC participants $(n=209)$ received usual follow-up services at the discretion of health departments. Basic nutrition and physical activity counseling pamphlets, pro- vided by the North Carolina Department of Health and Human Services, were available to MUC health departments. Participants in MSI $(n=302)$ were mailed six bimonthly computertailored health messages and received two telephone calls from health department staff.

The follow-up contacts were based on the assumption of a prior counseling relationship and were structured to efficiently reinforce the initial New Leaf . . . Choices for Healthy Living program. ${ }^{41}$ Intervention materials were based on social cognitive theory, ${ }^{44,45}$ relapse prevention theory, ${ }^{46}$ and the transtheoretical model (TTM). ${ }^{47}$ They were computer-tailored to each participant's behavioral goals, stage of change, knowledge, social support system, high-risk situations for relapse, and perceived benefits and barriers to behavior change and maintenance. The messages were designed for a low-literate, low-income adult population and featured testimonials and expert advice columns, feedback on participants' health behaviors, behavioral contracts and self-monitoring forms, social support cards, and health tips and quizzes. The intervention telephone calls were conducted by trained health department staff who assessed whether participants were meeting their goals and also helped participants to identify and negotiate barriers and set new goals. To facilitate counseling, staff members received phone call guides for each participant, which included such information as the participant's name, telephone number, behavioral goals, and perceived barriers.

On the basis of individual responses to the pretest survey, a separate set of materials was computer-generated for each participant. The material was mailed bimonthly and contained 32 messages selected specifically for each participant from a library of 649 messages. Similarly for the telephone calls, 10 messages were selected specifically for each participant from a library of 141 . A graphic design company produced the materials on a Mac OS computer using a program that matched participants' answers with specific messages and placed those messages in the final feedback form. The software used FileMaker Pro for data management, PageMaker for message and template management, and AppleScript for assembly and production functions.

\section{Phase Three: Evaluation measures}

Evaluation of health behaviors, beliefs, and attitudes was based on telephone surveys admin- 


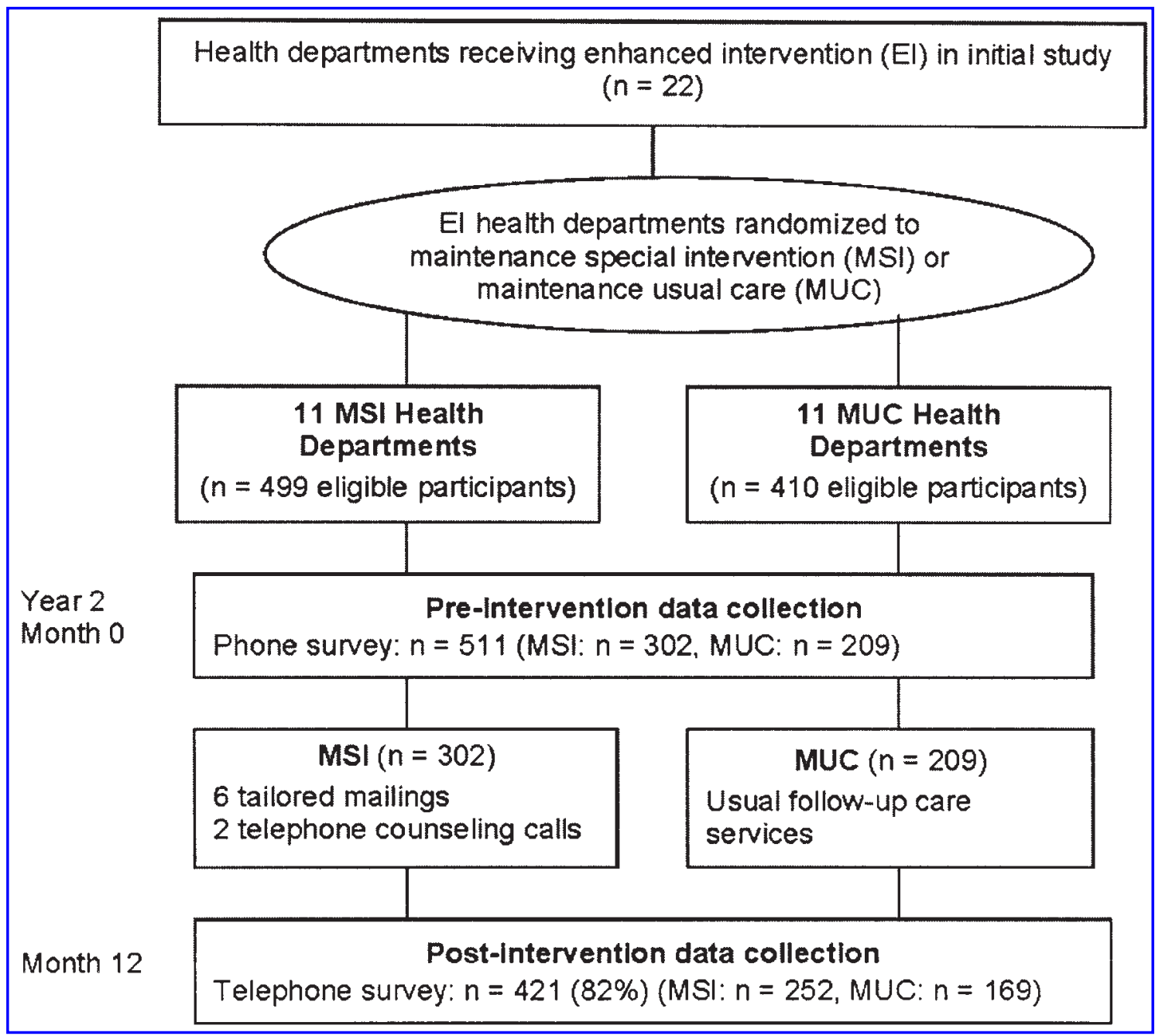

FIG. 1. Study design of the North Carolina WISEWOMAN maintenance program.

istered immediately before and 12 months after the maintenance intervention. The evaluation covered dietary intake, physical activity, stage of change, and selected psychosocial variables. We assessed program implementation and participant acceptability of the maintenance program in terms of recall, use, and satisfaction with the computer-tailored contacts, using data from the follow-up telephone survey and call logs recorded by health department staff on each participant's phone call guide.

Dietary intake. Dietary intake was assessed using the 54-item dietary risk assessment (DRA), ${ }^{48}$ a simplified, validated food frequency questionnaire that serves as a proxy measure for saturated fat and cholesterol intake. Validation of the DRA is described elsewhere. ${ }^{48}$
Physical activity. Physical activity was assessed using the 31-item physical activity assessment (PAA) questionnaire developed for the initial behavior change program. The PAA focuses on lifestyle activities relevant to midlife, low-income women and is designed to assess elements of sedentary and active lifestyles.

Stage of change. Using three questions based on the TTM, ${ }^{47}$ participants were categorized into stages at baseline: precontemplation (not thinking about change), contemplation (thinking about change within next 6 months), preparation (planning to change within next 30 days), action (currently trying to change), or maintenance (maintaining change for more than 6 months). The five categories were collapsed into three, defined by Kristal et al. ${ }^{49}$ as preaction (precontemplation, 
contemplation, or preparation), action, and maintenance.

Psychosocial variables. Self-efficacy to consume low-fat foods and be physically active was measured using one item on a 5 -point ( $1=$ very unsure, $5=$ very sure $)$ and 4 -point scale $(1=$ not at all sure, 4 = very sure), respectively. Beliefs about perceived barriers to eating low-fat foods were assessed using seven items on a 4-point scale ( $1=$ strongly agree, $4=$ strongly disagree), and perceived barriers to physical activity were assessed using nine items on a 3 -point scale $(1=$ a lot like me, 3 = not at all like me). Participants' motivation to eat well and be physically active were each measured using one item on a 4-point scale ( $1=$ not at all important, $4=$ very important). Perceived supportiveness of family and friends for eating healthy and being physically active was measured using one item for each behavior on a 4 -point scale ( $1=$ disagree a lot, $4=$ agree a lot). Responses were grouped into categories of low, medium, and high.

\section{Analysis}

Analyses are based on the 421 women who completed both surveys. Health departments were the unit of randomization, and participants were the unit of observation. To account for clustering among individuals within health departments, mixed models and generalized estimating equations (GEEs) were used to analyze continuous and categorical outcomes, respectively. Because we had a limited number of clusters, a modest number of observations per cluster, and several categories for each categorical variable, only main variables of interest were included in the models, while accounting for the clustering variable (health departments). Additional covariates, which would have overtaxed the models, were not included.

GEEs were used to examine intervention effects on health beliefs and attitudes using SAS PROC GENMOD (SAS Institute, Cary, NC, 2001). Models were constructed using the ordered categorical outcome as the dependent variable and study group as the main exposure, controlling for baseline status of the response variable and accounting for the clustering variable. Mixed models were used to assess change in DRA and PAA scores using SAS PROC MIXED. Models were constructed using change score as the dependent variable and intervention group as the main exposure variable, while incorporating a random intercept term to account for clustering. Using the standard of intent to treat, additional analyses were completed on all participants enrolled in the intervention $(n=511)$, assuming no change from baseline in persons without followup data $(n=90)$.

\section{RESULTS}

\section{Sample characteristics}

Of the 909 women eligible for the maintenance intervention, 511 (56\%) completed the pretest survey and enrolled in the follow-up program (302 in MSI and 209 in MUC) (Fig. 1). Reasons for nonresponse were refusals (8\%), no answer after 15 attempts $(10 \%)$, no telephone number provided $(2 \%)$, wrong number $(3 \%)$, disconnected telephone or no number listed (9\%), dropped from study (3\%), and special cases (e.g., language barriers, Alzheimer's disease, completed half the survey or less) $(9 \%)$. Nonrespondents $(n=398)$ did not differ significantly from respondents in age, race, education, or CVD risk factors assessed at baseline of NC WISEWOMAN, Phase Two.

Of the 511 participants who completed the pretest, $421(82 \%)$ were reinterviewed at the 12 month follow-up (Fig. 1). There were no significant differences between posttest respondents and nonrespondents by study arm, race, education, or baseline CVD risk factors. However, more slightly older women and women taking blood pressure medication at baseline $(p \leq 0.05)$ were in the follow-up group. Among the 90 women (18\%) unavailable for posttest, no differences were observed between the study groups in baseline demographics or CVD risk factors.

The mean age of participants was 59 years (Table 1). Forty-four percent were from ethnic minority groups, and only $8 \%$ had more than a high school education. Average total cholesterol and HDL cholesterol were $231 \mathrm{mg} / \mathrm{dl}$ and $53 \mathrm{mg} / \mathrm{dl}$, respectively, and mean SBP and DBP were 135 $\mathrm{mm} \mathrm{Hg}$ and $80 \mathrm{~mm} \mathrm{Hg}$, respectively. About half the women were obese, $16 \%$ smoked, $12 \%$ had a history of coronary heart disease (CHD), and 16\% had diabetes. Demographics and baseline risk factors were generally similar for both groups. 
Table 1. Baseline Demographics and Cardiovascular Disease

Risk Status of Maintenance Intervention Participants

\begin{tabular}{|c|c|c|c|}
\hline & $\begin{array}{c}\text { MSI } \\
(\mathrm{n}=302)\end{array}$ & $\begin{array}{c}\text { MUC } \\
(\mathrm{n}=209)\end{array}$ & $\begin{array}{l}\text { Total sample } \\
(\mathrm{n}=511)\end{array}$ \\
\hline \multicolumn{4}{|l|}{ Demographics } \\
\hline Age, years (mean) & 59 & 59 & 59 \\
\hline \multicolumn{4}{|l|}{ Education $(\%)^{\mathrm{a}}$} \\
\hline$<$ High school & 48 & 54 & 51 \\
\hline High school & 43 & 38 & 41 \\
\hline$>$ High school & 9 & 7 & 8 \\
\hline \multicolumn{4}{|l|}{ Race $(\%)^{\mathrm{b} *}$} \\
\hline Black & 38 & 43 & 40 \\
\hline White & 61 & 47 & 56 \\
\hline Native American or other & 1 & 9 & 4 \\
\hline \multicolumn{4}{|l|}{ CVD risk status ${ }^{\mathrm{C}}$} \\
\hline History of coronary heart disease $(\%)$ & 12 & 13 & 12 \\
\hline Smoker (\%) & 16 & 15 & 16 \\
\hline Diabetic (\%) & 16 & 17 & 16 \\
\hline Obese $(\% \text { BMI } \geq 30)^{d}$ & 48 & 57 & 52 \\
\hline Total cholesterol (mg/dl) & 234 & 228 & 231 \\
\hline HDL cholesterol $(\mathrm{mg} / \mathrm{dl})^{\mathrm{e}}$ & 53 & 52 & 53 \\
\hline Systolic blood pressure (mm Hg) & 135 & 134 & 135 \\
\hline Dastolic blood pressure (mm Hg) & 80 & 80 & 80 \\
\hline Taking BP medication (\%) & 44 & 50 & 47 \\
\hline
\end{tabular}

*Significant between-group differences at $p<0.05$.

aBased on $n=442$.

b Based on $n=487$.

'Based on $n=476$ (except where noted).

dBased on $n=472$.

eBased on $n=463$.

However, more MUC than MSI participants were from ethnic minority groups and obese $(p<0.05)$.

\section{Intervention effects}

Process measures. Ninety-six percent of MSI participants remembered receiving all six computertailored health mailings. Of these, 91\% reported reading all or most of the materials. Most (89\%) saved the mailings, but fewer $(51 \%)$ shared them with others. Almost all (97\%) were satisfied with the mailings, and most (76\%) thought the information was written especially for them.

On average, it took health department staff two attempts to reach participants by telephone, and each call lasted an average of 8 minutes. Among participants who recalled receiving a telephone call $(67 \%)$, almost all (94\%) were satisfied with the calls. Ninety-six percent thought the calls provided support for their behavior change efforts, and $92 \%$ felt the nurses understood a lot, or quite a bit, about their behavioral goals and barriers.

Dietary intake and physical activity level. Both groups of participants maintained low reported intake levels of dietary saturated fat and cholesterol (i.e., low DRA scores) (Table 2). At 12 months postintervention, the intervention effect for diet atherogenicity (posttest minus pretest score), adjusted for intrahealth department correlation, was $-0.39(p=0.60)$. No significant differences in PAA scores from preintervention to postintervention were found. Rather, both groups maintained somewhat low levels of physical activity (i.e., low PAA scores). At follow-up, the adjusted intervention effect for physical activity was $-0.25(p=0.62)$.

Additional analyses were completed on all participants enrolled in the follow-up intervention $(n=511)$, assuming no change from baseline for participants without follow-up data $(n=90)$. Adjusted for intrahealth department correlation, intervention effects at 12 months remained virtually unchanged (i.e., -0.32 [ $p=0.59]$ for diet atherogenicity and $-0.20[p=0.63]$ for physical activity).

Stage of change. Sixty percent or more of the women in each group had no change in stage. Most participants started and ended the 1-year program in the maintenance stage for both dietary 
Table 2. Effects of Maintenance Intervention on Dietary Risk and Physical Activity Scores $(n=421)^{\mathrm{a}}$

\begin{tabular}{|c|c|c|c|}
\hline & $\mathrm{n}$ & Baseline & $\begin{array}{l}\text { 12-month } \\
\text { follow-up }\end{array}$ \\
\hline \multicolumn{4}{|l|}{ Dietary risk assessment ${ }^{b}$} \\
\hline Maintenance special intervention $(\chi \pm \mathrm{SD})$ & 198 & $28.42 \pm 8.10$ & $28.19 \pm 7.12$ \\
\hline Maintenance usual care $(\chi \pm \mathrm{SD})$ & 136 & $29.52 \pm 7.87$ & $29.72 \pm 7.85$ \\
\hline $\begin{array}{l}\text { Intervention effect }{ }^{\mathrm{c}}(\beta \pm \mathrm{SE}) \\
p \text { value }\end{array}$ & & & $\begin{array}{c}-0.39 \pm 0.70 \\
0.60\end{array}$ \\
\hline \multicolumn{4}{|l|}{ Physical activity assessment ${ }^{\mathrm{d}}$} \\
\hline Maintenance special intervention $(\chi \pm \mathrm{SD})$ & 251 & $12.84 \pm 6.51$ & $12.86 \pm 6.69$ \\
\hline Maintenance usual care $(\chi \pm S D)$ & 165 & $12.68 \pm 5.96$ & $12.98 \pm 6.96$ \\
\hline Intervention $\operatorname{effect}^{\mathrm{c}}(\beta \pm \mathrm{SE})$ & & & $-0.25 \pm 0.49$ \\
\hline$p$ value & & & 0.62 \\
\hline
\end{tabular}

aparticipants completing pretest and posttest surveys.

bscore from 54-item scale, scored from 0 to 108 (not very to very atherogenic diet).

'Estimate for change score (posttest minus pretest score), adjusted for intrahealth department correlation (change scores calculated for participants with nonmissing scores at both pretest and posttest).

dScore from 31-item scale, scored from 0 to 42 (not very to very active).

and physical activity change (Tables 3 and 4). Forward movement was the next most common pattern (observed in 21\% of participants) and occurred most often between preaction and maintenance stages, followed by movement from action to maintenance. Backward movement was the least common pattern (observed in 14\% of participants) and mainly involved relapse from maintenance to preaction stages.

Adjusting for baseline levels and intrahealth department correlation, forward movement into later stages of dietary change did not differ significantly between study groups [OR $(95 \% \mathrm{CI})=$ $1.17(0.73,1.88),(p=0.51)$ ] (Table 3). However, intervention participants were significantly more likely than control participants to move forward into later stages of physical activity change [OR $(95 \%$ CI $)=1.65(1.07,2.56),(p=0.02)]$ (Table 4$)$.

Psychosocial variables. There were few changes in baseline levels over time in either group. Most participants began and ended the maintenance program with high levels of self-reported dietary and physical activity self-efficacy, social support, and motivation (Tables 3 and 4). Perceived barriers were low (physical activity) or moderate (lowfat eating). Some participants showed improvement in health beliefs and attitudes, but few regressed to lower than baseline levels.

Adjusting for baseline levels and intrahealth department correlation, shifts from less to more favorable levels of self-efficacy and social support for physical activity and motivation and perceived barriers to diet and physical activity did not differ significantly between groups $(p>0.10)$ (Tables 3 and 4). Differences were borderline significant for dietary self-efficacy and social support. Intervention participants were more likely to report increases in dietary self-efficacy [OR $(95 \% \mathrm{CI})=1.48(0.97,2.27), p=0.07]$, whereas control participants were more likely to report increases in dietary social support [OR $(95 \% \mathrm{CI})=$ $0.63(0.39,1.01), p=0.05]$.

\section{DISCUSSION}

To our knowledge, this project is the first one to use computer-tailored contacts as a posttreatment intervention strategy designed to sustain the effects of a more intensive intervention. Studies using computer-tailored communications have generally focused on adoption of health behaviors and suggest that tailored communications often, but not always, are associated with improvements in $\operatorname{diet}^{33-35,50-52}$ and physical activity $^{36,37,53}$ that can be reasonably well maintained for up to 6 months without further intervention. $^{39}$

We tested the effectiveness of computer-tailored messages in promoting sustained adherence to health-promoting behaviors. Computertailored mailed messages and telephone calls showed an advantage over usual follow-up care for promoting forward physical activity stage movement but did not appreciably affect other psychosocial or behavioral outcomes. Both groups (MSI and MUC) were able to maintain 
Table 3. Effects of Maintenance Intervention on Dietary Psychosocial Factors $(n=421)^{\mathrm{a}}$

\begin{tabular}{|c|c|c|c|c|c|c|}
\hline \multirow[b]{2}{*}{ Variable $^{\mathrm{b}}$} & \multicolumn{2}{|c|}{$M S I(\mathrm{n}=252)$} & \multicolumn{2}{|c|}{ MUC $(\mathrm{n}=169)$} & \multirow[b]{2}{*}{$\beta(S E)$} & \multirow[b]{2}{*}{ OR $(95 \% C I)$} \\
\hline & $\begin{array}{c}\text { Baseline } \\
\mathrm{n}(\%)^{\mathrm{c}}\end{array}$ & $\begin{array}{l}\text { Follow-up } \\
\mathrm{n}(\%)^{\mathrm{c}}\end{array}$ & $\begin{array}{l}\text { Baseline } \\
\mathrm{n}(\%)^{\mathrm{c}}\end{array}$ & $\begin{array}{c}\text { Follow-up } \\
\mathrm{n}(\%)^{\mathrm{c}}\end{array}$ & & \\
\hline \multicolumn{7}{|l|}{ Diet stage of change } \\
\hline Preaction & $72(30.4)$ & $53(22.4)$ & $58(36.7)$ & $45(28.5)$ & & \\
\hline Action & $20(8.4)$ & $14(5.9)$ & $14(8.9)$ & $6(3.8)$ & & \\
\hline Maintenance & 145 (61.2) & $170(71.7)$ & $86(54.4)$ & $107(67.7)$ & & \\
\hline Change estimate $^{\mathrm{d}}$ & & & & & $0.16(0.24)$ & $1.17(0.73,1.88)$ \\
\hline \multicolumn{7}{|l|}{ Diet social support } \\
\hline Low & $34(14.7)$ & $32(13.9)$ & $23(14.9)$ & $13(8.4)$ & & \\
\hline Medium & $37(16.0)$ & $41(17.8)$ & $29(18.8)$ & $27(17.5)$ & & \\
\hline High & $160(69.3)$ & $158(68.4)$ & $102(66.2)$ & $114(74.0)$ & & \\
\hline Change estimate ${ }^{\mathrm{d}}$ & & & & & $-0.47^{*}(0.24)$ & $0.63(0.39,1.01)$ \\
\hline \multicolumn{7}{|l|}{ Diet self-efficacy } \\
\hline Low & $26(10.4)$ & $18(7.2)$ & $20(11.9)$ & $24(14.3)$ & & \\
\hline Medium & $68(27.1)$ & $52(20.7)$ & $41(24.4)$ & 33 (19.6) & & \\
\hline High & $157(62.5)$ & $181(72.1)$ & $107(63.7)$ & $111(66.1)$ & & \\
\hline Change estimate ${ }^{\mathrm{d}}$ & & & & & $0.39 * *(0.22)$ & $1.48(0.97,2.27)$ \\
\hline \multicolumn{7}{|l|}{ Diet motivation } \\
\hline Low & $17(6.8)$ & $10(4.0)$ & $8(4.8)$ & $7(4.2)$ & & \\
\hline High & & $234(93.2)$ & $241(96.0)$ & $160(95.2)$ & $161(95.8)$ & \\
\hline Change estimate $\mathrm{d}$ & & & & & $0.12(0.51)$ & $1.12(0.41,3.06)$ \\
\hline \multicolumn{7}{|l|}{ Diet barriers } \\
\hline Low & 32 (17.1) & 31 (16.6) & $12(10.9)$ & $19(17.3)$ & & \\
\hline Medium & $130(69.5)$ & $138(73.8)$ & 79 (71.8) & 81 (73.6) & & \\
\hline High & $25(13.4)$ & $18(9.6)$ & 19 (17.3) & $10(9.1)$ & & \\
\hline Change estimate $\mathrm{d}$ & & & & & $-0.19(0.27)$ & $0.83(0.48,1.42)$ \\
\hline
\end{tabular}

aParticipants completing pretest and posttest surveys.

${ }^{\text {b}}$ Totals do not add up to 421 because of nonresponses to some items.

cPercentages may not add up to 100 because of rounding to the nearest tenth.

${ }^{\mathrm{d} C h a n g e ~ e s t i m a t e ~(r a t i o ~ o f ~ o d d s ~ o f ~ m o v i n g ~ f r o m ~ l e s s ~ t o ~ m o r e ~ f a v o r a b l e ~ o u t c o m e s ~ i n ~ M S I ~ v s . ~ M U C ~ g r o u p), ~ c o n-~}$ trolling for baseline level and intrahealth department correlation.

*Significant at the $p=0.05$ level.

$* *$ Borderline significant at the $p=0.07$ level.

positive dietary behaviors, health beliefs, and attitudes for up to 1 year after the NC WISEWOMAN project. Confidence in the validity of our findings is increased by the randomized study design and absence of differential attrition.

A limitation of this study is the use of self-report measures, which are susceptible to response set biases. ${ }^{54,55}$ In addition, although scores from the physical activity assessment compared favorably with Caltrac measures in a previous study $(r=0.36, p<0.0001),{ }^{56}$ the instrument has not been formally validated, which may result in errors in the measurement of physical activity. Another limitation is that participants who failed to complete the study may have had less favorable outcomes than those who completed it. This concern is mitigated to some extent by analyses of data on all participants who began the study; these analyses showed the same pattern of results. Another challenge to our interpretation of the study findings is the substantial loss to follow-up in the initial study (Phase Two), which limits the conclusions that can be drawn from the initial intervention. We conservatively defined "maintenance" as adherence to positive outcomes that may have already been present and not necessarily influenced by the initial intervention.

The failure of the intervention to demonstrate superior effects on many of the outcomes may be a result of insufficient strength of the maintenance intervention, a higher-than-expected effect of maintenance usual care (i.e., usual follow-up services at the discretion of health departments), a prolonged effect of the initial intervention, diminished power due to the group-assigned study design, measurement errors, or reduced precision caused by the small number of assignment units (health departments). Another explanation may relate to the observation that most participants began the maintenance program with low 
Table 4. Effects of Maintenance Intervention on Physical Activity Psychosocial Factors $(n=421)^{\mathrm{a}}$

\begin{tabular}{|c|c|c|c|c|c|c|}
\hline \multirow[b]{2}{*}{ Variable $^{\mathrm{b}}$} & \multicolumn{2}{|c|}{$M S I(\mathrm{n}=252)$} & \multicolumn{2}{|c|}{ MUC $(\mathrm{n}=169)$} & \multirow[b]{2}{*}{$\beta(S E)$} & \multirow[b]{2}{*}{ OR $(95 \% C I)$} \\
\hline & $\begin{array}{l}\text { Baseline } \\
\mathrm{n}(\%)^{\mathrm{c}}\end{array}$ & $\begin{array}{c}\text { Follow-up } \\
\mathrm{n}(\%)^{\mathrm{c}}\end{array}$ & $\begin{array}{l}\text { Baseline } \\
\mathrm{n}(\%)^{\mathrm{c}}\end{array}$ & $\begin{array}{c}\text { Follow-up } \\
\mathrm{n}(\%)^{\mathrm{c}}\end{array}$ & & \\
\hline \multicolumn{7}{|c|}{ Physical activity stage of change } \\
\hline Preaction & $63(26.4)$ & $56(23.4)$ & $35(23.3)$ & $47(31.3)$ & & \\
\hline Action & 30 (12.6) & $23(9.6)$ & $20(13.3)$ & 17 (11.3) & & \\
\hline Maintenance & $146(61.1)$ & $160(67.0)$ & $95(63.3)$ & $86(57.3)$ & & \\
\hline Change estimate ${ }^{\mathrm{d}}$ & & & & & $0.50 *(0.22)$ & $1.65(1.07,2.56)$ \\
\hline \multicolumn{7}{|c|}{ Physical activity social support } \\
\hline Low & $31(12.8)$ & $23(9.5)$ & $21(13.5)$ & $19(12.3)$ & & \\
\hline Medium & $50(20.7)$ & $28(11.6)$ & $26(16.8)$ & $21(13.6)$ & & \\
\hline High & $161(66.5)$ & $191(78.9)$ & $108(69.7)$ & $115(74.2)$ & & \\
\hline Change estimate $^{\mathrm{d}}$ & & & & & $0.32(0.25)$ & $1.38(0.85,2.25)$ \\
\hline \multicolumn{7}{|c|}{ Physical activity self-efficacy } \\
\hline Low & $58(23.2)$ & 54 (21.6) & $34(20.7)$ & $33(20.1)$ & & \\
\hline Medium & $84(33.6)$ & $77(30.8)$ & $59(36.0)$ & $51(31.1)$ & & \\
\hline High & $108(43.2)$ & 119 (47.6) & $71(43.3)$ & $80(48.8)$ & & \\
\hline Change estimate $^{\mathrm{d}}$ & & & & & $-0.05(0.20)$ & $0.95(0.65,1.40)$ \\
\hline \multicolumn{7}{|c|}{ Physical activity motivation } \\
\hline Low & $20(8.0)$ & $17(6.8)$ & $11(6.8)$ & $8(4.9)$ & & \\
\hline Medium & $24(9.6)$ & $20(8.0)$ & $7(4.3)$ & $16(9.9)$ & & \\
\hline High & 207 (82.5) & $214(85.3)$ & $144(88.9)$ & $138(85.2)$ & & \\
\hline Change estimate $^{\mathrm{d}}$ & & & & & $0.15(0.30)$ & $1.16(0.64,2.10)$ \\
\hline \multicolumn{7}{|c|}{ Physical activity barriers } \\
\hline Low & 118 (57.3) & $128(62.1)$ & $69(47.9)$ & $81(56.3)$ & & \\
\hline Medium & $70(34.0)$ & $62(30.1)$ & $63(43.8)$ & $46(31.9)$ & & \\
\hline High & $18(8.7)$ & $16(7.8)$ & $12(8.3)$ & 17 (11.8) & & \\
\hline Change estimate ${ }^{\mathrm{d}}$ & & & & & $0.15(0.24)$ & $1.16(0.73,1.86)$ \\
\hline
\end{tabular}

aparticipants completing pretest and posttest surveys.

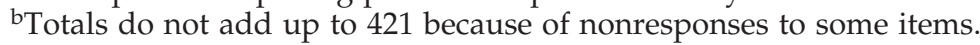

cPercentages may not add up to 100 because of rounding to the nearest tenth.

${ }^{\mathrm{d} C h a n g e ~ e s t i m a t e ~(r a t i o ~ o f ~ o d d s ~ o f ~ m o v i n g ~ f r o m ~ l e s s ~ t o ~ m o r e ~ f a v o r a b l e ~ o u t c o m e s ~ i n ~ M S I ~ v s . ~ M U C ~ g r o u p), ~}$ controlling for baseline level and intrahealth department correlation.

*Significant at the $p=0.02$ level.

reported levels of dietary risk and positive health beliefs and attitudes. This observation is not surprising considering that participants completed an intensive dietary and physical activity behavior change program ${ }^{41}$ prior to enrolling in the maintenance program, which may have created a ceiling effect and limited the amount of possible further gains in participants' self-reported dietary behavior, health beliefs, and attitudes. It is also possible that participants learned the correct responses to the assessment surveys after taking part in the initial program and completing the surveys previously. This could further explain the fact that they began the maintenance program reporting low dietary risk and positive health beliefs.

The NC WISEWOMAN project provided three half-hour counseling sessions over 6 months (initial intervention), followed by six mailings and two telephone calls (maintenance intervention).
These interventions may not have been potent enough to show significant effects over usual follow-up care in our sample of midlife, low-income women. Maintenance studies that have used more intensive behavior change programs (i.e., weekly, biweekly, or monthly contact) have produced significant improvements in $\operatorname{diet}^{28}$ and physical activity $18,19,23-25,31,32$ that were reasonably well maintained with frequent contacts in person, ${ }^{23,26}$ via mail or telephone, ${ }^{18,28,32}$ or using a combination of these approaches. ${ }^{17,19,24}$ However, most maintenance studies have recruited predominantly white, educated, middle-income adults, ${ }^{17,18,31,32}$ often selecting people who may be more motivated than the general population. ${ }^{19,23,26}$ Only one other study that we know of evaluated the effect of posttreatment contacts on maintenance of dietary behaviors in low-income, low-literate, multiethnic women. ${ }^{28}$ Frequent mail and telephone contacts were effective in promot- 
ing sustained dietary change following an intensive classroom-based intervention. ${ }^{28}$

Given the paucity of interventions targeting ethnically diverse, midlife, low-income women, further work refining maintenance interventions applicable to these populations is warranted. Future studies should also examine the effect of posttreatment tailored mail and telephone contacts on maintenance of behavior change following initial interventions of varying intensity. Issues related to the amount of maintenance intervention needed to be effective should also be addressed.

\section{ACKNOWLEDGMENTS}

We thank Thomas Keyserling, M.D., M.P.H., Beverly Garcia, M.P.H., Lisa Macon, B.S.P.H., B.A., and Nancy Aycock, M.S.W., all at the University of North Carolina at Chapel Hill's Center for Health Promotion and Disease Prevention. They were instrumental members of the study team. We also thank David Farrell, M.P.H., People Designs Inc., for producing the tailored materials; Ali Mokdad, Ph.D., Centers for Disease Control and Prevention, for his support and guidance; Larry Jenkins, M.P.H., Carolyn Townsend, M.P.H., R.N., and Pat Cannon, B.S.N., R.N., North Carolina Department of Health and Human Services, for their assistance in overseeing the field component of this study and the staff and participants at the 22 county health departments, whose generous cooperation made this project possible.

\section{REFERENCES}

1. American Heart Association. Heart and stroke statistical update, 2000. Dallas, TX: American Heart Association, 2001.

2. Winkleby MA, Kraemer HC, Ahn DK, et al. Ethnic and socioeconomic differences in cardiovascular disease risk factors: Findings from the Third National Health and Nutrition Examination Survey, 1988-1994. IAMA 1998;280:356.

3. Mansfield CJ, Wilson JL, Kobrinski EJ, et al. Premature mortality in the United States: The roles of geographic area, socioeconomic status, household type, and availability of medical care. Am J Public Health 1999;89:893.

4. Rosamond WD, Chambless LE, Folsom AR, et al. Trends in the incidence of myocardial infarction and in mortality due to coronary heart disease, 1987 to 1994. N Engl J Med 1998;339:861.
5. Krummel DA, Koffman DM, Bronner Y. Cardiovascular health interventions in women: What works? I Wom Health Gender Based Med 2001;10:117.

6. Miller TD, Balady GJ, Fletcher GF. Exercise and its role in the prevention and rehabilitation of cardiovascular disease. Ann Behav Med 1997;19:220.

7. NIH Consensus Development Panel on Physical Activity and Cardiovascular Health. NIH consensus conference: Physical activity and cardiovascular health. JAMA 1996;276:241.

8. Wylie-Rosett J, Swencionis C, Peters MH, et al. A weight-reduction intervention that optimizes use of practitioner's time, lowers glucose level, and raises HDL-cholesterol level in older adults. J Am Diet AsSoc 1994;94:37.

9. Svendsen OL, Hassager C, Christiansen C. Six months' follow-up on exercise added to a short-term diet in overweight postmenopausal women: Effects on body composition, resting metabolic rate, cardiovascular risk factors, and bone. Int J Obes Rel Metab Disord 1994;18:692.

10. Brownson RC, Smith CA, Pratt M, et al. Preventing cardiovascular disease through community-based risk reduction: The Bootheel Heart Health Project. Am J Public Health 1996;86:206.

11. Stevens VJ, Glasgow RE, Toobert DJ, et al. Randomized trial of a brief dietary intervention to decrease consumption of fat and increase consumption of fruits and vegetables. Am J Health Promot 2002;16:129.

12. Burke LE, Dunbar-Jacob JM, Hill MN. Compliance with cardiovascular disease prevention strategies: A review of the literature. Ann Behav Med 1997;19:239.

13. Orleans CT. Promoting the maintenance of health behavior change: Recommendations for the next generation of research and practice. Health Psychol 2000; 19(Suppl 1):76.

14. Pinto BM, Friedman R, Marcus BH, et al. Effects of a computer-based, telephone-counseling system on physical activity. Am J Prev Med 2002;23:113.

15. U.S. Department of Health and Human Services. Healthy people 2000: National health promotion and disease prevention objectives. Washington, DC: U.S. Government Printing Office, 1991.

16. Billings JH. Maintenance of behavior change in cardiorespiratory risk reduction: A clinical perspective from the Ornish Program for reversing coronary heart disease. Health Psychol 2000;19(Suppl 1):70.

17. King AC, Haskell WL, Young DR, et al. Long-term effects of varying intensities and formats of physical activity on participation rates, fitness, and lipoproteins in men and women aged 50 to 65 years. Circulation 1995;91:2596.

18. King AC, Frey-Hewitt BF, Dreon DM, et al. Diet vs exercise in weight maintenance. The effects of minimal intervention strategies on long-term outcomes in men. Arch Intern Med 1989;149:2741.

19. Brubaker PH, Rejeski WJ, Smith MJ, et al. A homebased maintenance exercise program after centerbased cardiac rehabilitation: Effects of blood lipids, body composition, and functional capacity. I Cardiopulm Rehabil 2000;20:50. 
20. Leermakers EA, Perri MG, Shigaki CL, et al. Effects of exercise-focused versus weight-focused maintenance programs on the management of obesity. Addict Behav 1999;24:219.

21. Perri MG, Martin AD, Leermakers EA, et al. Effects of group-versus home-based exercise in the treatment of obesity. I Consult Clin Psychol 1997;65:278.

22. Perri MG, Nezu AM, Patti ET, et al. Effect of length of treatment on weight loss. J Consult Clin Psychol 1989;57:450.

23. Clifford AP, Tan SY, Gorsuch RL. Efficacy of a selfdirected behavioral health change program: Weight, body composition, cardiovascular fitness, blood pressure, health risk, and psychosocial mediating variables. J Behav Med 1991;14:303.

24. Dunn AL, Marcus BH, Kampert JB, et al. Comparison of lifestyle and structured interventions to increase physical activity and cardiorespiratory fitness. JAMA 1999;281:327.

25. James LC, Folen RA, Page H, et al. The Tripler $L^{3} A N$ Program: A two-year follow-up report. Mil Med 1999; 164:389.

26. Lisspers J, Hofman-Bang C, Nordlander R, et al. Multifactorial evaluation of a program for lifestyle behavior change in rehabilitation and secondary prevention of coronary artery disease. Scand Cardiovasc I 1999;33:9.

27. Wilt S, Hubbard A, Thomas A. Knowledge, attitudes, treatment practices, and health behaviors of nurses regarding blood cholesterol and cardiovascular disease. Prev Med 1990;19:466.

28. Howard-Pitney B, Winkleby MA, Albright CL, et al. The Stanford Nutrition Action Program: A dietary fat intervention for low-literacy adults. Am J Public Health 1997;87:1971.

29. Heller RF, Knapp JC, Valenti LA, et al. Secondary prevention after acute myocardial infarction. Am J Cardiol 1993;72:759.

30. Haskell WL, Alderman EL, Fair JM, et al. Effects of intensive multiple risk factor reduction on coronary atherosclerosis and clinical cardiac events in men and women with coronary artery disease. Circulation 1994;89:975.

31. King AC, Haskell WL, Taylor CB, et al. Group- vs. home-based exercise training in healthy older men and women. JAMA 1991;266:1535.

32. King AC, Taylor CB, Haskell WL, et al. Strategies for increasing early adherence to and long-term maintenance of home-based exercise training in healthy middle-aged men and women. Am J Cardiol 1988;61:628.

33. Brug J, Steenhuis IHM, van Assema P, et al. The impact of a computer-tailored nutrition intervention. Prev Med 1996;25:236.

34. Brug J, Glanz K, van Assema P, et al. The impact of computer-tailored feedback and iterative feedback on fat, fruit and vegetable intake. Health Educ Behav 1998;25:357.

35. Campbell MK, DeVellis BM, Stretcher VJ, et al. Improving dietary behavior: The effectiveness of tailored messages in primary care settings. Am J Public Health 1994;84:783.
36. Bull FC, Kreuter MW, Scharff DP. Effects of tailored, personalized and general health messages on physical activity. Patient Educ Couns 1999;36:181.

37. Kreuter $\mathrm{MW}$, Strecher VJ. Do tailored behavior change messages enhance the effectiveness of health risk appraisal? Results from a randomized trial. Health Educ Res 1996;11:97.

38. Marcus BH, Bock BC, Pinto BM, et al. Efficacy of an individualized, motivationally tailored physical activity intervention. Ann Behav Med 1998;20:174.

39. Bock BC, Marcus BH, Pinto BM, et al. Maintenance of physical activity following an individualized motivationally tailored intervention. Ann Behav Med 2001; 23:79.

40. WISEWOMAN Workgroup. Cardiovascular disease prevention for women attending breast and cervical cancer screening programs: The WISEWOMAN projects. Prev Med 1999;28:496.

41. Ammerman A, Lutz S, Keyserling T, et al. A new leaf ... Choices for healthy living: Manual for assessment and counseling: Nutrition and physical activity. Chapel Hill, NC: University of North Carolina, 1998.

42. Rosamond WD, Ammerman AS, Holliday JL, et al. Cardiovascular disease risk factor intervention in lowincome women: The North Carolina WISEWOMAN project. Prev Med 2000;31:370.

43. Rosamond WR, Ammerman A, Tawney K, et al. Evaluation of the 1997-2000 North Carolina WISEWOMAN Project: Final report. Chapel Hill, NC: University of North Carolina Center for Health Promotion and Disease Prevention, 2001.

44. Bandura A. Self-efficacy: Toward a unifying theory of behavior change. Psychol Rev 1977;84:191.

45. Bandura A. Social foundations of thought and action: A social cognitive theory. Englewood Cliffs, NJ: Prentice-Hall, 1986.

46. Marlatt GA, George WH. Relapse prevention: Introduction and overview of the model. Br J Addict 1984; 79:261.

47. Prochaska JO, DiClemente CC. Stages and processes of self-change in smoking: Toward an integrative model of change. I Consult Clin Psychol 1983;51:390.

48. Ammerman A, Haines P, DeVellis R, et al. A brief dietary assessment to guide cholesterol reduction in low-income individuals: Design and validation. I Am Diet Assoc 1991;91:1385.

49. Kristal AR, Glanz K, Tilley BC, Li S. Mediating factors in dietary change: Understanding the impact of a worksite nutrition intervention. Health Educ Behav 2000;27:112.

50. Campbell MK, Bernhardt JM, Waldmiller M, et al. Varying the message source in computer-tailored nutrition education. Patient Educ Couns 1999;36:157.

51. Brug J, Steenhuis IHM, van Assema P, et al. Computer-tailored nutrition education: Differences between two interventions. Health Educ Res 1999;14:249.

52. Campbell MK, Tessaro I, DeVellis B, et al. Effects of a tailored health promotion program for female bluecollar workers: Health Works for Women. Prev Med 2002;34:313.

53. Marcus BH, Emmons KM, Simkin-Silverman LR, et 
al. Evaluation of motivationally tailored vs. standard self-help physical activity interventions at the workplace. Am J Health Promot 1998;12:246.

54. Kristal AR, Andrilla HA, Koepsell TD, et al. Dietary assessment instruments are susceptible to intervention-associated response set biases. I Am Diet Assoc 1998;98:40.

55. Hebert JR, Ma Y, Clemow L, et al. Gender differences in social desirability and social approval bias in dietary self-report. Am J Epidemiol 1997;146:1046.

56. Keyserling TC, Samuel-Hodge CD, Ammerman AS, et al. A randomized trial of an intervention to improve self-care behaviors of African-American women with type 2 diabetes: impact on physical activity. Diabetes Care 2002;25:1576.

Address reprint requests to: Joan Kavanagh UNC-CH 1700 Airport Road Campus Box 8140 Chapel Hill, NC 27599-8140

E-mail: Joan_Kavanagh@unc.edu 


\section{This article has been cited by:}

1. Ann DeSmet, Dimitri Van Ryckeghem, Sofie Compernolle, Tom Baranowski, Debbe Thompson, Geert Crombez, Karolien Poels, Wendy Van Lippevelde, Sara Bastiaensens, Katrien Van Cleemput, Heidi Vandebosch, Ilse De Bourdeaudhuij. 2014. A meta-analysis of serious digital games for healthy lifestyle promotion. Preventive Medicine 69, 95-107. [CrossRef]

2. V. Cleland, K. Ball. 2013. What might work? Exploring the perceived feasibility of strategies to promote physical activity among women living in socioeconomically disadvantaged neighbourhoods. Health Education Research 28, 205-219. [CrossRef]

3. V. Cleland, A. Granados, D. Crawford, T. Winzenberg, K. Ball. 2013. Effectiveness of interventions to promote physical activity among socioeconomically disadvantaged women: a systematic review and metaanalysis. Obesity Reviews 14:10.1111/obr.2013.14.issue-3, 197-212. [CrossRef]

4. Karen Broekhuizen, Willemieke Kroeze, Mireille NM Poppel, Anke Oenema, Johannes Brug. 2012. A Systematic Review of Randomized Controlled Trials on the Effectiveness of Computer-Tailored Physical Activity and Dietary Behavior Promotion Programs: an Update. Annals of Behavioral Medicine 44, 259-286. [CrossRef]

5. Maureen Reni Courtney, Scott E. Conard, Pat Dunn, Kristen Scarborough. 2011. The Game of Health@: An innovative lifestyle change program implemented in a family practice. Journal of the American Academy of Nurse Practitioners 23, 289-297. [CrossRef]

6. Daniela N Schulz, Stef PJ Kremers, Liesbeth ADM van Osch, Francine Schneider, Mathieu JG van Adrichem, Hein de Vries. 2011. Testing a Dutch web-based tailored lifestyle programme among adults: a study protocol. BMC Public Health 11, 108. [CrossRef]

7. Karen Glanz, Donald B. Bishop. 2010. The Role of Behavioral Science Theory in Development and Implementation of Public Health Interventions. Annual Review of Public Health 31, 399-418. [CrossRef]

8. Kathleen Smith-DiJulio, Debra Anderson. 2009. Sustainability of a Multimodal Intervention to Promote Lifestyle Factors Associated With the Prevention of Cardiovascular Disease in Midlife Australian Women: A 5-year Follow-Up. Health Care for Women International 30, 1111-1130. [CrossRef]

9. Jodi M. Cahill, Jeanne H. Freeland-Graves, Bijal S. Shah, Hongxing Lu, Deborah M. Klohe-Lehman. 2009. Development and Validation of the Eating Stimulus Index in Low-Income, Minority Women in Early Postpartum. Journal of the American Dietetic Association 109, 1593-1598. [CrossRef]

10. Pouran D. Faghri, Erika Blozie, Sara Gustavesen, Rajashree Kotejoshyer. 2008. The Role of Tailored Consultation Following Health-Risk Appraisals in Employees' Health Behavior. Journal of Occupational and Environmental Medicine 50, 1378-1385. [CrossRef]

11. Kim D. Reynolds, David B. Buller, Amy L. Yaroch, Julie Maloy, Cristy R. Geno, Gary R. Cutter. 2008. Effects of Program Exposure and Engagement With Tailored Prevention Communication on Sun Protection by Young Adolescents. Journal of Health Communication 13, 619-636. [CrossRef]

12. Jo Ellen Vrazel, Ruth P. Saunders, Sara Wilcox. 2008. An Overview and Proposed Framework of SocialEnvironmental Influences on the Physical-Activity Behavior of Women. American Journal of Health Promotion 23, 2-12. [CrossRef]

13. H. de Vries, S. P. J. Kremers, T. Smeets, J. Brug, K. Eijmael. 2008. The Effectiveness of Tailored Feedback and Action Plans in an Intervention Addressing Multiple Health Behaviors. American Journal of Health Promotion 22, 417-425. [CrossRef]

14. Neera Chaudhary, Nancy Kreiger. 2007. Nutrition and Physical Activity Interventions For Low-Income Populations. Canadian Journal of Dietetic Practice and Research 68, 201-206. [CrossRef]

15. Gregory J. Norman, Marion F. Zabinski, Marc A. Adams, Dori E. Rosenberg, Amy L. Yaroch, Audie A. Atienza. 2007. A Review of eHealth Interventions for Physical Activity and Dietary Behavior Change. American Journal of Preventive Medicine 33, 336-345.e16. [CrossRef] 
16. T. Smeets, S. P. J. Kremers, H. Vries, J. Brug. 2007. Effects of tailored feedback on multiple health behaviors. Annals of Behavioral Medicine 33, 117-123. [CrossRef]

17. G. Sorensen, A. M. Stoddard, T. Dubowitz, E. M. Barbeau, J. Bigby, K. M. Emmons, L. F. Berkman, K. E. Peterson. 2007. The Influence of Social Context on Changes in Fruit and Vegetable Consumption: Results of the Healthy Directions Studies. American Journal of Public Health 97, 1216-1227. [CrossRef]

18. Claire I. Viadro. 2004. Taking Stock of WISEWOMAN. Journal of Women's Health 13:5, 480-483. [Citation] [Full Text PDF] [Full Text PDF with Links]

19. Antronette K. Yancey. 2004. Building Capacity to Prevent and Control Chronic Disease in Underserved Communities: Expanding the Wisdom of WISEWOMAN in Intervening at the Environmental Level. Journal of Women's Health 13:5, 644-649. [Citation] [Full Text PDF] [Full Text PDF with Links] 